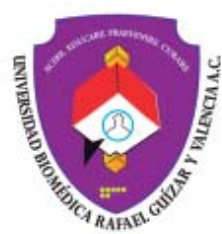

Vol. 8, Núm. 2

\title{
Tratamiento de la parálisis facial periférica con ácido alfa lipoico $[A A L]$ más reentrenamiento muscular
}

\author{
Treatment of peripheral facial paralysis with alpha lipoic acid (ALA) plus muscle retraining
}

José Reyes Sánchez Domínguez, ${ }^{*}$ Ricardo Manzur Lorenzo Velasco, ${ }^{\ddagger}$ Francisco Aguilar Rebolledo

\section{RESUMEN}

Antecedentes: Hasta el momento no existe un tratamiento eficaz para los pacientes con parálisis facial periférica (PFP) crónica. Objetivo: Se llevó a cabo un estudio prospectivo controlado para examinar la eficacia del reentrenamiento muscular de la PFP, además de la administración de ácido alfa lipoico oral. Material y métodos: Pacientes de casos: se incluyeron 18 pacientes con parálisis facial subaguda o crónica (al menos de un mes a varios años), ocho con grado $\mathrm{V}$ según la clasificación de House Brackmann, seis con grado IV y cuatro con grado III. Pacientes control: fueron siete pacientes, cuatro con grado IV por clasificación de House Brackmann $\mathrm{y}$ tres con grado III, funcionaron como controles recibiendo tratamiento convencional: vitaminas y masajes. Comorbilidades: 10 pacientes con diabetes mellitus (DM2), ocho hipertensión combinado HTA y DM2, 12 pacientes con una evolución media de dos años y uno a ocho años de recorrido, hemoglobina glucosilada con promedio de $7.5 \%$ y de 6.5 a $10.9 \%$. Todos fueron tratados con ácido alfa lipoico a dosis de $600 \mathrm{mg}$ diarios durante scuatro meses. Y además de reentrenamiento muscular, músculo por músculo. Resultados: Casos: ocho pacientes de grado V descendieron a grado III, después de dos meses de tratamiento, alcanzaron la recuperación completa a los cuatro meses. Seis con grado IV y cuatro con grado III se

\section{ABSTRACT}

Introduction: So far there is no effective treatment for patients with chronic peripheral facial paralysis (PFP). Objective: A prospective controlled study was conducted to examine the efficacy of PFP muscle retraining in addition to oral alpha-lipoic acid administration. Material and methods: Case patients: 18 patients with subacute or chronic facial paralysis (at least one month to several years), eight patients with grade $V$ according to the House Brackmann classification, six patients with grade IV and four patients with grade III were included. Control patients: seven patients, four patients with grade IV by House Brackmann classification, three patients with grade III functioned as controls receiving conventional treatment: vitamins and massages. Comorbidities: 10 patients with diabetes mellitus (DM2), hypertension, eight patients combined HBP and DM2. 12 patients with a mean evolution of two years and one to eight years of experience, glycated hemoglobin with $\bar{x}$ of 7.5 and 6.5 to $10.9 \%$. All patients were treated with alpha lipoic acid at a dose of $600 \mathrm{mg}$ daily for four months. And in addition to muscle retraining, muscle by muscle. Results: Cases: eight patients from grade V descended to grade III, after two months of treatment, they achieved complete recovery at four months. Six patients with grade IV and four

* Director de la Clínica de Rehabilitación y Terapia Física, México.

* Licenciado en Terapia Física y Rehabilitación, Clínica CIMA, México.

§ Rector de la Universidad Biomédica Rafael Guízar y Valencia (UNIBIO), Xalapa, Ver.

Correspondencia:

Dr. José Reyes Sánchez Domínguez

E-mail: sanchezdominguezjr@hotmail.com

Recibido: 22-09-2021. Aceptado: 25-10-2021.

Citar como: Sánchez DJR, Lorenzo VRM, Aguilar RF. Tratamiento de la parálisis facial periférica con ácido alfa lipoico (AAL) más reentrenamiento muscular. Plast Restaur Neurol. 2021;8 (2): 66-72. https://dx.doi.org/10.35366/103084 
recuperaron ad integrum a los dos meses. Los pacientes control con tratamiento convencional, bajaron un punto del grado IV a los dos meses y otro punto más hasta los seis meses y su recuperación casi total se alcanzó hasta el año. Estadística, con alta significancia a un nivel de $0.5(\mathrm{p}<0.05)$. Especialmente en la medición de la expresión facial, comparando casos y control $(p<0.05)$. Conclusiones: Estos resultados demuestran que el reentrenamiento muscular (terapia de masaje músculo por músculo) para la parálisis del nervio facial periférico, incluida la retroalimentación en espejo en combinación con ácido alfa lipoico, es un tratamiento clínicamente eficaz en casos con parálisis facial subaguda y crónica.

Palabras clave: Parálisis de Bell, ácido alfa lipoico, programa de reentrenamiento muscular. patients with grade III recovered ad integrum after two months. Control patients with conventional treatment dropped one point from grade IV at two months and another point at six months, and their almost total recovery was achieved up to one year. Statistics. With high significance at a level of $0.5(p<0.05)$. Especially in the measurement of facial expression, comparing cases and controls $(p<0.05)$. Conclusions: These results demonstrate that muscle retraining (muscle-by-muscle massage therapy) for peripheral facial nerve palsy, including mirror feedback in combination with alpha lipoic acid, is a clinically effective treatment in patients with subacute and chronic facial paralysis.

Keywords: Bell's palsy, alpha lipoic acid, muscle retraining program.

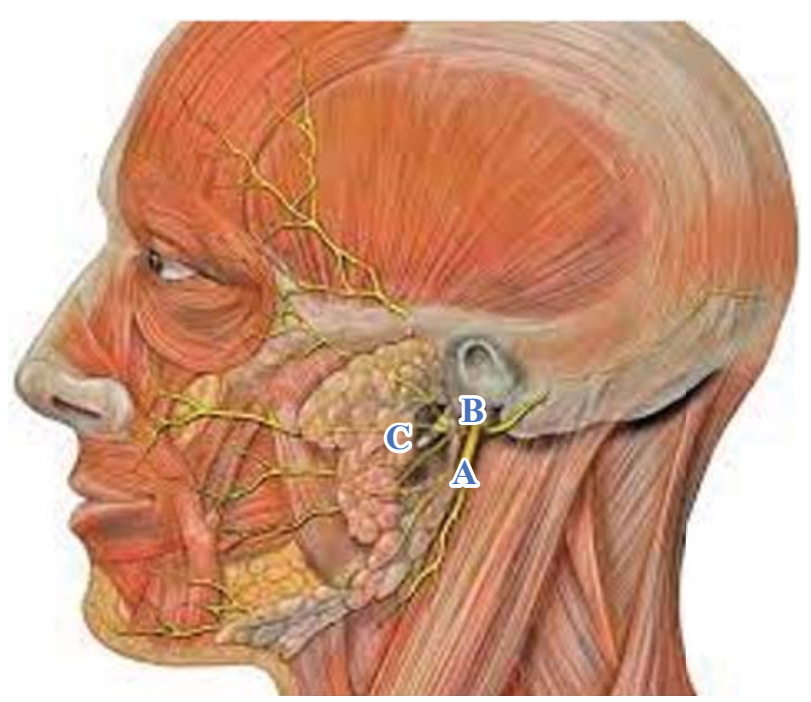

Figura 1: Nervio facial. A-C) Señalan lesiones del nervio en el orificio estilo mastoideo, en sentido distal y proximal al ganglio geniculado, respectivamente. Así como también cada una de las estructuras involucradas en la patogénesis de la parálisis facial.

En estudios experimentales del reentrenamiento muscular se ha demostrado su utilidad incrementando la supervivencia de neuronas en situaciones de daño, o ayudando a mantener la integridad del axón en caso de lesiones focales. Técnicas de electroneurografía demuestran que después de este tiempo el potencial evocado supramáximo pierde su excitabilidad, y esto puede reflejar una degeneración progresiva en la vaina de mielina del nervio. ${ }^{9,10}$

El objetivo de este trabajo es la evaluación del reentrenamiento selectivo del control muscular combinado con la administración de ácido alfa lipoico (AAL), un antioxidante capaz de regenerar el proceso de desmie- 
linización por daño isquémico por inflamación del proceso lesional en el nervio facial.

\section{MATERIAL Y MÉTODOS}

Se estudió un grupo de 18 casos con parálisis facial periférica (PFP), 10 con diabetes mellitus tipo 2 (DM2), ocho con hipertensión arterial, ambas con una evolución de siete años, recorrido de tres a 15 años. La hemoglobina glucosilada (HbA1c) fue en promedio de $7.5 \%$ y un recorrido de 6.5 a $10.9 \%$. Todos reunieron los siguientes criterios de inclusión y siete controles que recibieron el tratamiento convencional, multivitamínicos y estimulación electromecánica.

\section{Casos:}

1. Tiempo de evolución mayor de un mes de inicio de la parálisis.

2. No recibir ningún tratamiento médico especializado (esteroides).

3. PFP sin etiología sintomática (tumores, neurofibromas o traumática).

4. TAC de Cráneo normal para todos los pacientes.

5. La etiología probable de carácter idiopático (probablemente viral).
6. Todos alcanzaron un control de su $\mathrm{HbA} 1 \mathrm{c}$ de $7 \%$ durante el tratamiento.

Ingresaron al estudio 10 mujeres y ocho hombres con media de edad de $47 \pm 7.8$ años y con un recorrido de 24 a 60 años.

\section{Controles:}

1. Tiempo de evolución mayor de un mes de inicio de la parálisis.

2. Recibir tratamiento fisioterápico.

3. El tratamiento que recibieron fue el convencional a base de vitamínicos y terapia tradicional, la mayoría fueron foráneos y ya estaban recibiendo dicho tratamiento.

4. El sexo de los pacientes; cuatro mujeres y tres hombres.

5. La media de edad de los controles $46 \pm 7.8$ años con un recorrido de 30 a 60 años.

Para ambos grupos se usaron las mismas escalas de evaluación.

\section{Estudios a realizar en todo paciente con parálisis facial}

Historia clínica. Debe realizarse una historia clínica completa con su respectivo examen físico detallado.

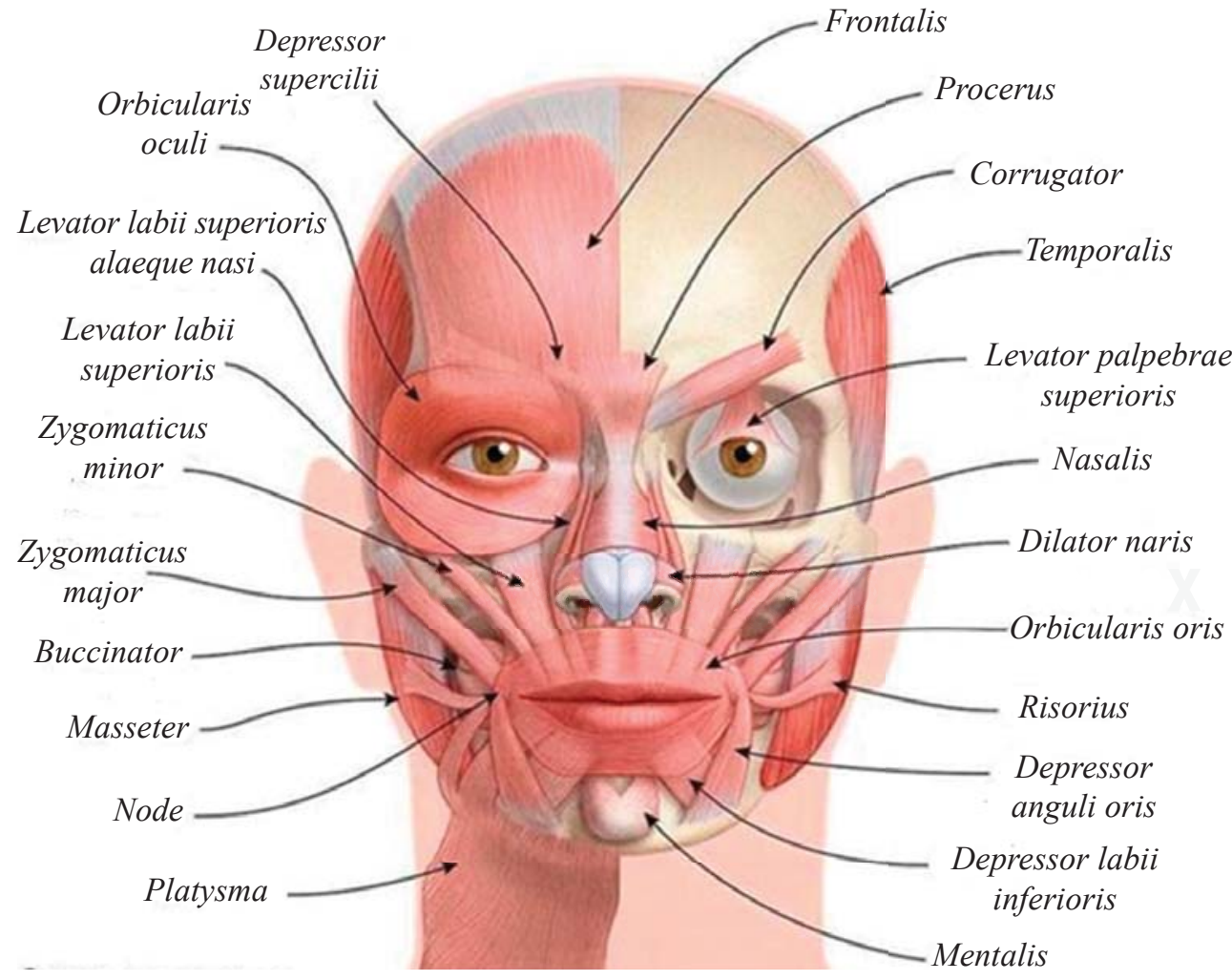

Figura 2:

Representación esquemática de los músculos de la expresión facial. 
Evaluando cada una de las acciones de los músculos faciales, tono, simetría y función motora. A nivel sensorial evolución de la intensidad del sonido, secreción lagrimal y sensación del gusto para café, tabaco y azúcar.

La presión arterial registrada tanto en posición sentada como en decúbito mostró cifras dentro de límites normales para ambos grupos.

Otoscopia. En todos los pacientes, casos y controles demostró un conducto auditivo externo normal.

Neuroimagen. A todos se les realizó tomografía computarizada de cráneo o resonancia magnética sin identificar patología del tallo cerebral o lesiones tumorales cerca de la emergencia del nervio facial en la unión ponto bulbar (ángulo ponto cerebeloso).

Exámenes de laboratorio. A todos los pacientes, casos y controles se les realizó biometría hemática completa y química sanguínea, descartando alteraciones hematológicas y las alteraciones del metabolismo de los hidratos de carbono (diabetes mellitus tipo 2 -DM2-) en los 10 casos con glucemia de 160 a $310 \mathrm{mg} / \mathrm{dL}$, ocho pacientes con hipertensión arterial y DM2; llegando el control de $7 \%$ en los cuatro meses siguientes, observando mejor control de la dieta, ejercicio y cambio de hipoglucemiantes, 11 pacientes con sobrepeso, seis con colesterol $260 \mathrm{mg}$. Seis con triglicéridos elevados $210 \mathrm{mg} / \mathrm{mL}$.

Los controles cuatro pacientes con DM2 con glucosa de $210 \mathrm{mg} / \mathrm{mL}$. La hemoglobina A1c promedio de $7.7 \%$ y llegando al control en los cuatro meses siguientes, ordenando su dieta, estimulando el ejercicio y cambio de hipoglucemiantes. Cuatro pacientes con sobrepeso.

Estudios neurofisiológicos. La electromiografía (EMG) mostró potenciales de fibrilación en los músculos frontalis y zygomaticus, lo cual corrobora la pérdida de la inervación.

El reflejo del parpadeo es un examen de naturaleza polisináptica donde se transmite un estímulo unilateral por vía del nervio trigémino ( $V$ nervio craneal), el cual produce una respuesta motora temprana en el nervio facial ipsilateral, seguido por una respuesta bilateral tardía (músculo del estapedio contralateral). Para estimular dicho reflejo, se usó la estimulación eléctrica del nervio supraorbitario en lugar de aire sobre la córnea. El registro se hizo colocando electrodos sobre ambos bordes infraorbitarios. Las respuestas tardías fueron retardadas en $40 \%$ de los casos y ausentes en $60 \%$.

Es necesario mencionar que la evaluación de la función del nervio facial brinda la posibilidad de identificar signos o síntomas atípicos que requieren la referencia urgente al especialista o a la realización de un estudio de imagen.
Audiograma. Mostró hipoacusia en $70 \%$ de los casos y en $20 \%$ de los controles.

\section{Tipo de tratamiento fisioterapéutico}

Reentrenamiento muscular (músculo por músculo) de los músculos afectados por la parálisis del nervio facial: se divide la estimulación en los segmentos oftálmico (frontalis, corrugator, procerus, orbicularis oculi, cigomático mayor y menor, nasal lateral, nasal del ala de la nariz), maxilar superior y mandibular. Terminando la estimulación, se incluyó retroalimentación frente al espejo, muecas o gesticulaciones durante cinco a 10 minutos.

Terapia física: terapia de control y estimulación selectiva (Figura 2) (músculo por músculo-Balliet y colaboradores). Cada tercer día se hace una reevaluación y nueva evaluación para poder clasificar la parálisis y dar un valor del porcentaje de mejoría.

Programa de reentrenamiento muscular selectivo y retroalimentación con espejo: está basado en la observación detallada de la disfunción de la musculatura de la cara, la simetría y función sensitiva del nervio facial (VII par) que como resultado de daño al nervio se ha manifestado, dicha alteración se puede modificar desde vías de comportamiento motor y sensitivo del sistema nervioso central, ya que el reentrenamiento motor tiene un importante componente sensitivo, de hecho es importante pensar que la recuperación de uno de los músculos no será única, sino que compartirá la recuperación (plasticidad) del otro músculo asociado en cadena muscular, es decir, sin atender el otro.

\section{Tratamiento no farmacológico}

En todos los pacientes, independientemente del grado en el cual se clasifiquen, deberá iniciarse tratamiento básico, que consta de cuatro aspectos fundamentales:

Protección ocular: uso de lentes oscuros con protección lateral, para evitar la irritación por rayos solares, efecto traumático del polvo y prevenir la lesión corneal por desecación.

Lubricación ocular: mediante ungüento oftálmico (sólo por la noche) y uso de lágrimas artificiales (metilcelulosa o hipromelosa, ambas una gota cada una o dos horas por dos semanas).

Oclusión ocular nocturna: mediante parche oclusor y férula bucal para provocar estiramiento de la comisura labial afectada.

Evitar el lavado ocular frecuente de té de manzanilla, ya que puede propiciar procesos alérgicos, usarlo con delicadeza y suave. 
Tabla 1: Escala de valoración de House Brackmann para evaluar el grado de afección, los cuales se clasificaron en la siguiente manera:

\begin{tabular}{ccc}
\hline Grado & Descripción & Características \\
\hline I & Normal & Función normal en todas las áreas \\
II & Disfunción leve & Debilidad muscular notoria solo en la exploración \\
III & Disfunción moderada & Deficiencia obvia, pero no desfigurante entre ambos lados de la cara \\
IV & Disfunción moderadamente severa & Asimetría desfigurante \\
V & Disfunción severa & Escaso movimiento perceptible \\
VI & Parálisis total & Sin movimiento \\
\hline
\end{tabular}

\section{Tratamiento fisioterapéutico}

Se aplica calor local con compresa húmeda-caliente por 10 minutos a hemicara afectada, para mejorar la circulación.

Respecto a la electroterapia, la evidencia es controversial sobre su uso y efectividad en la recuperación de la parálisis facial periférica, sobre todo en la etapa inicial. En el caso de la parálisis facial crónica sí se recomienda en la etapa tardía (después de varios meses).

Las investigaciones básicas sugieren la posibilidad de aumento de reinervación anómala con su uso, lo que se considera poco probable ya que se estimula el punto motor del músculo y no el nervio. Además, la estimulación no es retrógrada, es decir, si se estimula el músculo tendría que atravesar la unión mioneural o placa neuromuscular para llegar en forma retrógrada al nervio, lo cual no es factible.

A la fecha, las revisiones sistematizadas se encuentran en fase de protocolo, por lo que se sugiere el juicio clínico para la aplicación de este recurso en músculos paralizados o con mínima contracción visible o palpable, por punto motor y no en masa, sólo para mantener el trofismo muscular. Se recomienda realizar reeducación muscular y retroalimentación.

La reeducación muscular supervisada y frente al espejo son la piedra angular del tratamiento rehabilitatorio, las cuales están organizadas en cuatro etapas de acuerdo con los hallazgos físicos a la exploración y la etapa en que se encuentra el paciente. Las principales acciones van encaminadas a lograr el correcto funcionamiento de la oclusión del ojo y de la boca.

La etapa inicial se caracteriza por asimetría en reposo, movimientos mínimos voluntarios, ausencia de sincinesias y daño funcional grado IV y $V$ según la clasificación de House Brackmann, se realizarán los siguientes ejercicios asistidos:

1. El paciente se auxilia por el dedo índice y medio colocado sobre el músculo a trabajar en dirección al movimiento deseado, sosteniéndolos por unos segundos en hemicara afectada.

2. Hacer énfasis en realizarlos simétricamente respecto a hemicara sana y en forma aislada con el resto de los músculos de hemicara afectada, dándole mayor importancia a los músculos orbicular de los párpados y labios, ya que son los últimos en recuperarse.

3. Levantar con los dedos la ceja involucrada y sostenerla por unos segundos.

4. La asistencia manual disminuirá progresivamente de acuerdo con la recuperación muscular.

Educación al paciente. Informar sobre características de su patología y alternativas de tratamiento y diagnóstico, a fin de disminuir la ansiedad generada por la patología.

El uso de la goma de mascar (chicle para masticar) y de vibradores mecánicos no se recomienda, ya que puede provocar fatiga muscular por acción global de la musculatura facial y de los músculos de la masticación, sobre todo cuando la debilidad es grave grado IV y $\mathrm{V}$.

Ejercicios activos en el lado afectado: se retira la presión digital y se solicita trabajar en el siguiente orden: frontal, superciliar, elevador del labio superior, canino, buccinador, borla y cuadrado de la barba, triangular de los labios, cutáneo del cuello y por último los cigomáticos.

Ejercicios activos en el lado sano: la contractura de los músculos sanos, por parálisis del lado contralateral ocasiona aumento del tono, trastornos de la simetría y función motora, por lo que también debe de tratarse.

\section{Terapia farmacológica}

Los pacientes seleccionados para el estudio no recibieron esteroides tanto en forma intravenosa, intramuscular o por vía oral, $30 \%$ de los pacientes tanto de los casos como de los controles recibieron diversas dosis de vitamínicos fundamentalmente vitamina B. 


\section{Medicamento administrado a pacientes de casos}

Régimen: ácido alfa lipoico (AAL), sólo 600 mg diarios, y combinado L-Carnitina 1,000 mg o combinado con gamalinolénico $400 \mu \mathrm{g}$ cada 24 horas y durante los alimentos.

Los pacientes con parálisis facial subaguda (más de un mes hasta años de iniciada) franca fueron invitados a este estudio abierto. Los pacientes estaban libres de analgésicos y 10 de ellos eran diabéticos. Comenzaron con ácido alfa lipoico (ALA) 600 mg durante ocho semanas con mejoría clínica significativa de los síntomas. El efecto antioxidante el ácido alfa lipoico mejora la mielinización del nervio periférico. A causa de que disminuye el estrés oxidativo, también ayuda en las enfermedades neurodegenerativas como el Alzheimer, Parkinson y demencia.

Al ingresar al estudio, los pacientes se clasifican de acuerdo a la escala de valoración de House Brackmann (Tabla 1).

Casos $(\mathrm{N}=18)$ :

1. Ocho pacientes grado $\mathrm{V}$

2. Seis pacientes grado IV

3. Cuatro pacientes grado III

Controles $(N=7)$ :

1. Cuatro pacientes grado IV

2. Tres pacientes grado III

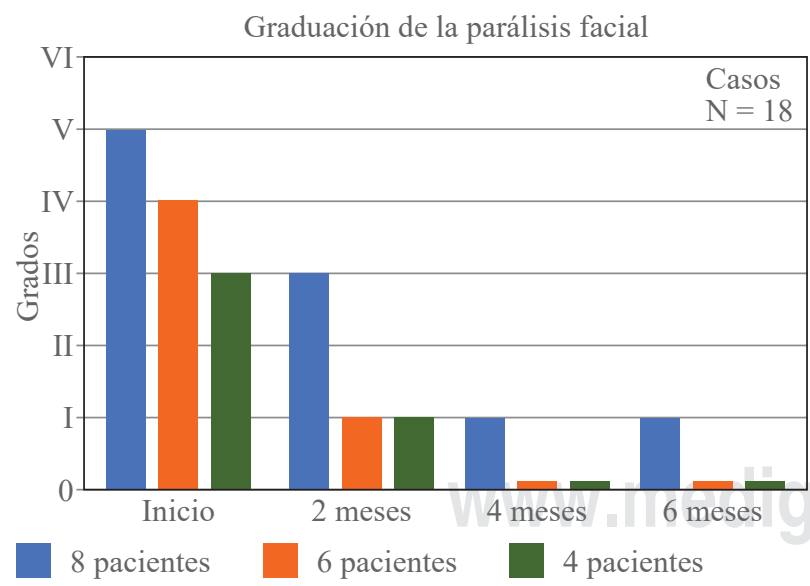

Figura 3: Casos pacientes: Se observa que el grupo de los 8 pacientes de inicio grado $\mathrm{V}$ (barra azul) mejoraron con el tratamiento a los dos meses a grado III y a los cuatro meses remisión completa grado I. El grupo de los 6 pacientes iniciaron grado IV (barra color naranja) mejorando a los dos meses a grado I. El grupo de los 4 pacientes que iniciaron grado III (barra color verde) mejoraron a los dos meses a grado I. En contraste con el grupo control a una significancia de $5 \%$ con una $\mathrm{p}<0.05$ (significativa).

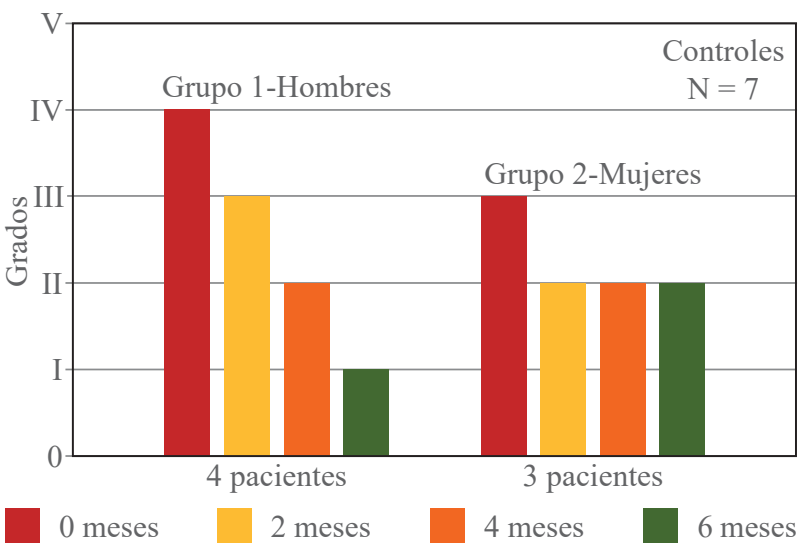

Figura 4: Casos controles: Cuatro pacientes correspondientes al grupo 1 iniciaron en grado IV (barra roja), observando a los 2 meses una mejoría a grado III (barra color naranja), a los cuatro meses sólo bajaron un punto grado II (barra naranja), logrando remisión completa grado I hasta los 6 meses (barra verde). Los siguientes 3 pacientes del grupo 2 iniciaron con grado III y sólo se observó mejoría a grado II hasta los 6 meses de seguimiento.

\section{RESULTADOS}

En los pacientes del grupo tratado (casos) $(\mathrm{N}=18)$, ocho fueron mujeres y 10 hombres con una media de edad $47 \pm 8.7$ años. Los pacientes controles fueron siete, de los cuales tres eran mujeres y cuatro hombres con una media de edad de $46 \pm 7.8$ años.

Casos: a los dos meses los ocho pacientes con grado $\mathrm{V}$ bajaron a grado III y se recuperaron en su totalidad hasta el cuarto mes (grado I-normal). Todos fueron tratados con AAL más reentrenamiento muscular y gesticulaciones con espejo y se curaron antes de los cuatro meses, tal y como muestran las Figuras 3 y 4.

\section{DISCUSIÓN}

El tratamiento de la parálisis facial idiopática es controversial. El uso de esteroides en etapas tempranas en el curso de la enfermedad se ha sugerido como una manera de reducir la inflamación existente del nervio y en consecuencia la duración de la parálisis. ${ }^{10,11}$

El tratamiento parece ser más efectivo cuando se inicia de manera temprana dentro de las primeras 24 horas de iniciada la parálisis. Algunos virus, entre ellos herpes simple y varicela zóster, han sido implicados en la patogénesis de la parálisis facial idiopática y en razón de ello, se ha sugerido de agentes antivirales. ${ }^{11}$

Un gran número de medidas terapéuticas se han reportado en la PFP, tales como: la vitamina B1, B2, 
B6, B12, solas o en combinación con esteroides con resultados controversiales. El oxígeno hiperbárico puede mejorar la parálisis facial idiopática. ${ }^{8,12}$

En casos de debilidad facial de más de un mes de evolución considerada de subaguda a crónica persistente, uno de los tratamientos es la intervención cosmética, ya que las implicaciones de tipo psicosocial son importantes para los pacientes. ${ }^{6}$ Los programas de ejercicio con reentrenamiento muscular han mostrado beneficio en aquéllos con parálisis facial de larga evolución, y si este programa se asocia con retroalimentación con espejo, la posibilidad de mejoría aumenta. El ácido alfa lipoico (AAL) se ha asociado con la proliferación capilar, crecimiento neural y formación de sinapsis. Por otra parte, en estudios experimentales con daño neurológico inducido por hipoxia/isquemia, el AAL ha demostrado mejorar la función neuronal en varias investigaciones clínicas. La recuperación de la función puede estar mediada por estímulos hacia conexiones sinápticas y probablemente arborizaciones axónicas que han cambiado potencialmente los patrones alterados de la actividad neuronal.

Por otro lado, la recuperación de la función también puede darse por la sustitución de impulsos sensoriales con nuevos programas generados por la regeneración de la mielina que recubre al nervio, estimulada en gran medida por AAL (neuroplasticidad). ${ }^{11,12}$

\section{CONCLUSIONES}

Estos resultados sugieren que una rehabilitación apropiada más un programa con reentrenamiento muscular, es decir estimulación músculo por músculo, es conveniente para potenciar la mejoría, promoviendo la regeneración. En el caso de asociar con un antioxidante que favorece la plasticidad de la fibra nerviosa y la mielina, se promueve que el impulso nervioso sea corregido de manera temprana.

Este tratamiento de AAL más reentrenamiento muscular puede conducir a una recuperación de la lesión después de daño neurológico, en la parálisis facial periférica idiopática.

Multivitamínicos y esteroides pueden ser de utilidad en etapas muy tempranas de la parálisis. En la PFP la combinación de medicamento AAL y el programa de reentrenamiento muscular (músculo por músculo) demostraron ser útiles en mejorar la función en este grupo de pacientes.
Las recomendaciones de los autores sugieren en caso de parálisis facial enviar al paciente a un especialista en rehabilitación y terapia física, neurología, nutrición, oxígeno hiperbárico sin olvidar el reentrenamiento muscular por parte del paciente. Como en la clínica Universitaria CIMA de Xalapa, donde integra todos los servicios.

\section{REFERENCIAS}

1. Zeng L, Alongkronrusmee D, van Rijn RM. An integrated perspective on diabetic, alcoholic, and drug-induced neuropathy, etiology, and treatment in the US. J Pain Res. 2017;10:219-228.

2. Juster-Switlyk K, Smith AG. Updates in diabetic peripheral neuropathy. F1000Res. 2016;5:F1000 Faculty Rev-738. doi: 10.12688/f1000research.7898.1.

3. Bromberg MB. Peripheral neurotoxic disorders. Neurol Clin. 2000;18(3):681-894.

4. Goetz CG, Pappert EJ. Textbook of Clinical Neurology. Philadelphia: WB Saunders Co; 1999.

5. Pourmand R. Diabetic neuropathy. Neurol Clin. 1997;15(3):569-576.

6. Sugimoto K, Murakawa Y, Sima AA. Diabetic neuropathy--a continuing enigma. Diabetes Metab Res Rev. 2010;16(6):408-433.

7. Zochodne DW. Diabetic polyneuropathy: an update. Curr Opin Neurol. 2008;21(5):527-533.

8. Casas-Rodera P, Lassaletta L, González T, Sarriá MJ, Gavilán J. Técnicas reconstructivas del nervio facial. Acta Otorrinolaringológica Española. 2007;58(4):133-137. doi: 10.1016/ S0001-6519(07)74898-7.

9. Gastol J, Kapusta P, Polus A et al. Epigenetic mechanism in search for the pathomechanism of diabetic neuropathy development in diabetes mellitus type 1 (T1DM). Endocrine. 2020;68(1):235-240.

10. Altaf QA, Ali A, Piya MK, Raymond NT, Tahrani AA. The relationship between obstructive sleep apnea and intra-epidermal nerve fiber density, PARP activation and foot ulceration in patients with type 2 diabetes. J Diabetes Complications. 2016;30(7):1315-1320.

11. Balliet R, Shinn JB, Bach-y-Rita P. Facial paralysis rehabilitation: retraining selective muscle control. Int Rehabil Med. 1982;4(2):67-74.

12. Perkins BA, Olaleye D, Bril V. Carpal tunnel syndrome in patients with diabetic polyneuropathy. Diabetes Care. 2002;25(3):565-569.

Declaración de ética: Se cumplieron los requerimientos éticos. Los participantes proporcionaron su consentimiento informado por escrito para participar en este estudio.

Financiamiento: Este estudio no fue apoyado por subvenciones.

Conflicto de intereses: Los autores declaran que la investigación se llevó a cabo en ausencia de relaciones comerciales o financieras que pudieran interpretarse como un posible conflicto de intereses. 